\title{
Da Vinci $S$ robotic surgery in the treatment of benign and malignant gynecologic tumors
}

\author{
Grigor Gortchev • Slavcho Tomov • \\ Latchesar Tantchev • Angelika Velkova • \\ Zdravka Radionova
}

Received: 29 September 2009 / Accepted: 22 October 2009/Published online: 5 December 2009

(C) Springer-Verlag 2009

\begin{abstract}
The objective of this work is to present and analyze our da Vinci $S$ robotic surgery results in the treatment of gynecologic tumors for a 1-year period. Fiftyone patients underwent da Vinci S (Intuitive Surgical, USA) robotic surgery at the Medical University-Pleven Gynecologic Oncology Clinic from January 2008 to January 2009. Robot-assisted radical hysterectomy with total pelvic lymph node dissection was performed in $28(54.9 \%)$ of them, robot-assisted total hysterectomy in $21(41.2 \%)$, and robot-assisted pelvic lymph node dissection in two (3.9\%). The average length of the operative intervention, from the beginning of the $\mathrm{CO}_{2}$ insufflation of the abdomen to closing trocar skin incision was $119.89 \min ( \pm 43.441)$ and mean console time was $76.56 \mathrm{~min}( \pm 32.904)$. The average patient body weight was $70.56 \mathrm{~kg}( \pm 18.272$; range, 41$114)$ with mean body mass index (BMI) of $27.30( \pm 6.938)$. No significant difference was observed between the BMI and operative time $(p=0.49)$. No significant intra-operative complications were registered. An ureterovaginal fistula was diagnosed on the 10th postoperative day in one of the patients $(2 \%)$. Robot-assisted endoscopic gynecologic surgery is a modern and advanced method for operative treatment of benign and malignant gynecological tumors. It is appropriate for obese patients as the obesity is not related to a prolonged operative time.
\end{abstract}

Keywords Da Vinci S robotic system .

Robot-assisted hysterectomy · Benign gynecologic tumors . Malignant gynecologic tumors

G. Gortchev $(\bowtie) \cdot$ S. Tomov $\cdot$ L. Tantchev $\cdot$ A. Velkova •

Z. Radionova

Gynecologic Oncology Clinic, Medical University Pleven,

Pleven, Bulgaria

e-mail: oncogynec@yahoo.com

\section{Introduction}

Over the last decade, the efforts of a number of surgeons and engineers to find a scientifically valid technologic solution of the problems related to the development of laparoscopic and minimally invasive surgery have been combined. The robotic surgical systems play the central role in their projects. Robotically assisted surgical interventions overcome many of the shortcomings of classical laparoscopy, such as the limitation of four degrees of freedom of the instruments and 2D vision [1].

The Food and Drug Administration (USA) approved the use of the da Vinci robotic system for performing intraabdominal surgical procedures in 2000 [2]. The first application of the computer-enhanced telemanipulator in gynecological practice was introduced to perform a microsurgical tubal reanastomosis [3]. Indications for surgery expand, and the application of the da Vinci robotic system in the field of gynecologic surgery for performing simple hysterectomy, myomectomy, and radical hysterectomy with lymph node dissection have gradually been increased [4-6].

The objective of this article is to analyze our da Vinci $\mathrm{S}$ robotic surgery results in the treatment of benign and malignant gynecologic tumors for a 1-year period, presenting some specific aspects of our protocol.

\section{Materials and methods}

Fifty-one patients underwent the da Vinci S (Intuitive Surgical, Sunnyvale, CA, USA) robotic surgery at the Medical University_Pleven Gynecologic Oncology Clinic from January 2008 to January 2009. Robot-assisted radical hysterectomy with total pelvic lymph node dissection was performed in $28(54.9 \%)$ of them, robot-assisted total 
simple hysterectomies in $21(41.2 \%)$, and robot-assisted pelvic lymph node dissections in two (3.9\%). The operating team, consisting of a console surgeon, patient-side assistant, and patient-side nurse, were trained and certified to work with a robot in the European training center IRCAD_EITS in Strasbourg.

Determinant excluding criteria in patient selection for robotic surgery were as follows: advanced cervical and endometrial carcinoma, American Society of Anesthesiologists class $\geq \mathrm{III}$, and preoperative radio/chemotherapy.

The da Vinci S robotic system, which we use, is composed of three major components: a surgeon console, a patient cart, and a vision cart. The surgeon's console is the monitoring center of the system. The robotic surgeon operates from the console out of the sterile field and controls the instruments and the three-dimensional camera with his hands and feet, using two major hand controls and foot pedals. The apparatus for monopolar and bipolar coagulation is connected to the panel of the pedal switch (Autocon II 400, Storz). The patient cart is the operative component of the da Vinci S system. It is composed of four arms (Endo Wrist technology): three arms for controlling the surgical instruments and one arm for the camera and endoscope. The operator of the patient cart works in the sterile surgical field. The source of cold light, modules for camera control, a focus controlling mechanism, an intercom system, and a touch screen are located on the vision cart. The non-sterile person works with the appliances on the visual cart but the touch screen is dressed in a sterile cover and allows the patient-side assistant and patient-side nurse to operate with it.

In the operative interventions, the following Endo Wrist instruments (Intuitive Surgical) were used: Maryland Bipolar Forceps in the first robotic arm, Monopolar Curved Scissors at the second arm, and Cadier Forceps at the third arm. On putting stitches in the vaginal cuff, the bipolar forceps and monopolar scissors were replaced with suture cut needle driver and large needle driver, respectively. Ultracision Harmonic Scalpel (Ethicon Endo Surgery) and Harmonic Curved Shears (Intuitive Surgical) were used in part of the surgical interventions.

\section{Description of our protocol}

\section{Operating room set-up}

The operating room where the da Vinci S surgical system is located was equipped in the pattern of Operating Room 1 (OR1; Storz). It has a local and central control center-Storz Communication Bus interface with a possibility for coordination and using the entire system of appliances, as well as for videoconference connection, virtual consultations, and training. OR1 is an open system, which guarantees the optimal using and integration of innovative technologies, such as the da Vinci S surgical system.

Before getting the patient into the operating room the scrub nurse prepares two instrument tables-one for abdominal and the other for vaginal manipulations, and after that puts on the robotic arms in sterile sacks. The situation of the da Vinci S surgical system components is consistent with the instructions of the Intuitive Surgical, Sunnyvale, CA, USA and the specific conditions available in OR1. The operating table with the patient plays a central role. The surgeon's console is located on the left side of the patient. The patient's side cart is disposed so that after the relevant preparation to be placed in between the patient's legs as quickly as possible. The patient-side assistant and patient-side nurse are on the right side of the operating table and close to them, the vision cart is placed, allowing monitors' and touch screen control. Upon getting in the operating room, the patent is placed in the gynecologic position (dorsal lithotomy) and after being introduced to general anaesthesia, the operating table is moved down maximally, so that the patient is placed in Trendelenburg position $\left(30^{\circ}-45^{\circ}\right)$. Before the $\mathrm{CO}_{2}$ insufflation, a second assistant places uterine-manipulator (Clermont-Ferrand, Storz) and Foley catheter. The $\mathrm{CO}_{2}$ insufflation is accomplished with the Veress needle, and in cases suspicious for adhesions, the Optical Veress with Miniature Straight Forward Telescope $0^{\circ}$, diameter $2 \mathrm{~mm}$, length $20 \mathrm{~cm}$ (Storz) is used.

\section{Port placement}

According to the recommendations of the Intuitive Surgical, the port for the camera must be placed at 10 $20 \mathrm{~cm}$ away from the target structure. We position it on the medial line about 5-7 $\mathrm{cm}$ above the umbilical level. We use a 12-mm trocar (Ethicon Endo Surgery), placed without or under optic control (Optical Veress). The remaining ports placements are as follows:

- Port for the first robotic working arm- $-8-12 \mathrm{~cm}$ on the right and $15^{\circ}-30^{\circ}$ distally from the camera port, trocar $8 \mathrm{~mm}$;

- Port for the second robotic working arm-8-12 $\mathrm{cm}$ on the left and $15^{\circ}-30^{\circ}$ distally from the camera port, trocar $8 \mathrm{~mm}$;

- Port for the third robotic working arm-8-12 $\mathrm{cm}$ laterally from the port for the second working arm, trocar $8 \mathrm{~mm}$;

- Port 1 for assistance-8-12 cm laterally from the port for the first working arm, trocar $5 \mathrm{~mm}$;

- Port 2 for assistance $-8-10 \mathrm{~cm}$ distally from port 1 for assistance, trocar $10 \mathrm{~mm}$. 


\section{Operative technique}

Robotically assisted radical hysterectomy, which we perform, corresponds to class III extended hysterectomy according to Piver et al. [7]. It includes consecutively the following phases:

- Total pelvic lymph node dissection bilaterally (Fig. 1);

- Disconnection of the ovarian vessel's bundle;

- Clasping and cutting of the uterine artery immediately after its separation from the internal iliac artery;

- Opening of the vesicouterine fold and dissection of the urinary bladder from the uterine cervix and the upper vagina;

- Dissection of the ureter from the vesicouterine ligament to its infusion into the urinary bladder;

- Opening of the peritoneum of cavum Douglasi and dissection of the anterior rectal wall from the posterior uterine arch and uterine wall;

- Transection of the uterosacral ligaments at the level of the anterior rectal wall;

- Transection of the lateral parametrium maximally close to the pelvic wall;

- Circular transection of the vagina;

- Continuous suture of the vaginal cuff with 2-0 Vicryl $15 \mathrm{~cm}$ long (Ethicon Endo Surgery).

The patient-side assistant helps for better exploration of the operative field, places hemostatic clasps, performs irrigation and aspiration and passes needles and sutures during the surgical intervention. The second assistant stands upright in between the patient's legs and the patient cart. His role is subsidiary but extremely important for the visualization of the anatomical structures in adequate positioning of the uterus.

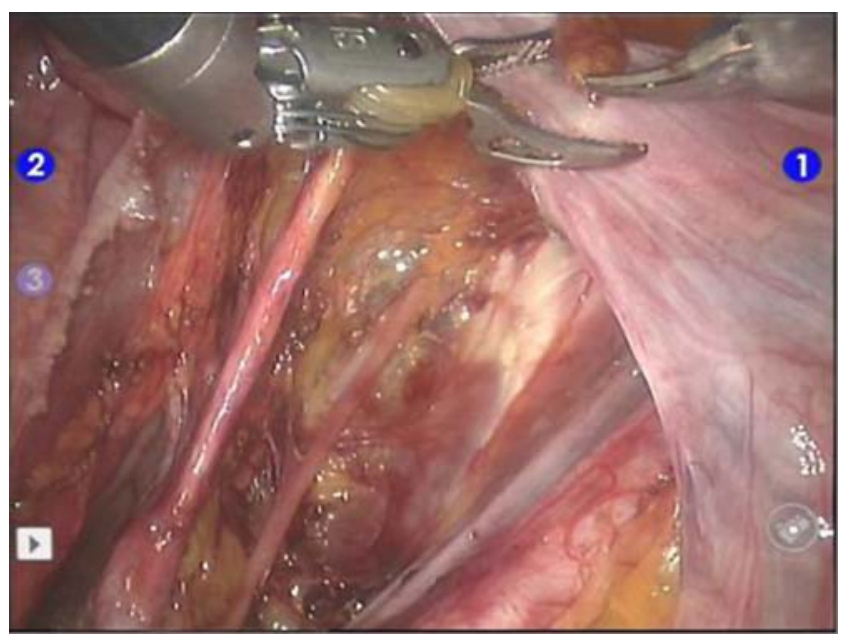

Fig. 1 Obturator fossa on the right side: status post total pelvic lymph dissection
The stages of the robotically assisted total hysterectomy, which we perform, do not differ from those of the classic total laparoscopic hysterectomy. The use of the uterinemanipulator cup after the dissection of the urinary bladder from the uterine cervix and coagulation of the uterine arteries, facilitate significantly and reduce the time of the operative intervention.

\section{Results}

Mean patients' age was 48.56 years $( \pm 10.675)$, as the predominant age group varied between 40 and 49 years $(n=21 ; 41.2 \%)$. The main clinical characteristics are presented in Table 1 .

According to the tumor histology, the patients were distributed as follows: with planocellular cervical carcinoma, 22 (43.1\%); endocervical adenocarcinoma, five (9.8\%); endometroid adenocarcinoma of the uterus, six $(11.8 \%)$; serous papilliferous adenocarcinoma of the endometrium, two (3.9\%); cervical carcinoma in situ, three $(5.9 \%)$; uterine leiomyoma, seven (13.7\%); adenomyosis uteri, three $(5.9 \%)$; and others, three $(5.9 \%)$. The mean length of the operative intervention, from the beginning of the $\mathrm{CO}_{2}$ insufflation of the abdomen to closing trocar skin incision, (incision time-skin closed) was $119.89 \mathrm{~min}( \pm 43.441), 145 \mathrm{~min}( \pm 36.619)$ for the robotically assisted radical hysterectomies, and $98.18 \mathrm{~min}$ $( \pm 28.266)$ for the robotically assisted total simple hysterectomies, respectively ( $p=0.002)$.

The mean console time for all operative interventions was $76.56 \mathrm{~min}( \pm 32.904), 95.93 \mathrm{~min}( \pm 29.298)$ for the robotically assisted radical hysterectomies, and $55.82 \mathrm{~min}$

Table 1 Clinical characteristics

\begin{tabular}{ll}
\hline Characteristics & Value \\
\hline Body weight $(\mathrm{kg})$ & \\
$\quad$ Range & $41-114$ \\
Mean & 70 \\
Height $(\mathrm{cm})$ & \\
Range & $150-175$ \\
Mean & 159.93 \\
BMI & \\
Range & $17-43$ \\
Mean & 27.18 \\
Indication for surgery $(n)$ & \\
Cervical carcinoma & $27(52.9 \%)$ \\
Carcinoma of the uterus & $8(15.8 \%)$ \\
Uterine myoma & $7(13.7 \%)$ \\
Others & $9(17.6 \%)$ \\
\hline
\end{tabular}

$B M I$ body mass index; $n$ number of patients 
$( \pm 24.248)$ for the robotically assisted total simple hysterectomies, respectively $(p=0.012)$.

Table 2 presents all time intervals, reported during our work with the robot.

The effective blood loss (EBL) was investigated $(50 \mathrm{~mL} \pm 6.486)$, as well as the mean hematocrit values during the preoperative $(0.38 \pm 0.048)$ and postoperative period (on the 1st postoperative day; $0.34 \pm 0.045$ ), and a significant difference was observed between the last two variables $(p=0.001)$.

Significant difference was observed in the robotically assisted radical hysterectomies as well as in the robotically assisted total simple hysterectomies $(p=0.001)$.

The average number of the removed pelvic lymph nodes was $11.36( \pm 2.285)$. No regional lymph metastases were found in the histological investigation

No significant differences were observed between the mean body mass index (BMI) and the mean time needed for the total pelvic lymph node dissection $(p=0.63)$, between the mean BMI and incision time-skin closed $(p=0.49)$, and the mean console time $(p=0.40)$, respectively.

No significant difference was observed between EBL and BMI $(p=0.211)$.

No significant intra-operative complications were registered. An ureterovaginal fistula was diagnosed on the 10th postoperative day in one of the patients $(2 \%)$.

\section{Discussion}

The $\mathrm{CO}_{2}$ insufflation of the abdomen, correct positioning and trocars placement are the key points for successfully performed robotically assisted surgery. Inadequate pneumoperitoneum is the leading cause for bad exploration and visibility in the operative field, which reduces the work effectiveness and elongates the operative time. Seamon

Table 2 Time characteristics of different phases of robot-assisted surgical interventions

\begin{tabular}{lc}
\hline Time characteristics & Mean time min \\
\hline Time in room-out of room & $171.75( \pm \mathrm{SD} 44.471)$ \\
Incision time-skin closed & $119.89( \pm \mathrm{SD} 43.441)$ \\
Docked time & $5.63( \pm \mathrm{Std}$. Error 0.765$)$ \\
System docked-undocked & $96.07( \pm \mathrm{SD} 38.934)$ \\
Console time & $76.56( \pm \mathrm{SD} 32.904)$ \\
Lymph nodes right & $16.86( \pm \mathrm{SD} 5.260)$ \\
Lymph nodes left & $19.80( \pm \mathrm{SD} 6.109)$ \\
Lymph nodes (total) & $36.07( \pm \mathrm{SD} 10.101)$ \\
Colpotomy & $9.79( \pm \mathrm{SD} 4.791)$ \\
Vaginal cuff & $12.13( \pm \mathrm{SD} 4.928)$ \\
\hline
\end{tabular}

et al. use an open technique. Only in extremely obese patients, the peritoneum is entered with the Veress needle [1]. Our experience, achieved in the classic laparoscopy, allows us with a high degree of confidence to use the technique with the Veress needle for creating pneumoperitoneum, in patients with obesity and/or previous laparotomias we insufflate $\mathrm{CO}_{2}$ and place all trocars with the help of optical Veress.

The position of ports must be chosen, minding the type of the operative intervention (radical or simple hysterectomy), the specific anatomy of each patient, and the type of instruments, which are to be used.

The meaning of the correct trocar placement is to avoid collision of the arms of the patient cart and to expand maximally the limits of instruments and endoscope movement. Seamon et al. use six ports - four robotic ports and two accessory ports. They place the $5-\mathrm{mm}$ port for assistance beneath the costal margin, at least $5 \mathrm{~cm}$ above and between the camera and robotic port 1 [1]. Different placements of two of the ports are found in the protocol of Marchal et al. They use four ports, placing a 12-mm trocar for the endoscope and the camera at the umbilical level and a $10-\mathrm{mm}$ trocar for the assistant's hand-several centimeters above the connecting line between the ports of the camera and the left working robotic arm [8]. We place six ports in the robotically assisted radical hysterectomies (four working and two accessory), while in the robotically assisted total hysterectomies we place four ports (three working and one $10-\mathrm{mm}$ accessory). The different placement of the accessory ports in our protocol is consistent with the recommendations of Intuitive Surgical and under the specific conditions in the operating room type OR1, allows optimal operation with the da Vinci S robotic system.

The report for the first robotic-assisted radical hysterectomy, accomplished by Sert and Abeler, was published in 2006 [6]. Several articles, which analyze a great number of cases were published later: Magrina and Zanagnolo, 27 patients and Bogges et al., 51 patients $[9,10]$. Our team performed its first robot-assisted radical hysterectomy in May 2008 on a 54-year-old patient with cervical carcinoma, stage IB1. Until January 2009, 27 more radical hysterectomies with pelvic lymph node dissections followed, as 26 of them were in-patients with cervical carcinoma (IB stage), and one-with endometrial carcinoma (IIA stage). In the lymph node dissections, we strictly keep the principle for lymph node removal "en block" (external iliac, internal iliac, and obturator lymph nodes). External iliac vessels are mobilized from $\mathrm{m}$. iliopsoas, fossa lumbosacralis is opened and the remaining lymph tissue around the obturator nerve and truncus lumbosacralis is removed for maximum oncologic safety achievement. Lymph nodes are placed in an endobag and evacuated through working $10-\mathrm{mm}$ trocar. 
One of the basic stages in the surgery is the ureter's dissection. It is identified on the posterior sheet of plica lata right after its transition over the iliac vessel bundle. It is traced to the level of its crossing with the uterine artery. Extremely important is the skeletonization of artery in " $T$ ", branch of the uterine artery, which supplies the ureter. Selective coagulation and cutting of this artery allows the release of the ureter from the uterine vessel bundle and the adjacent to it lymph tissue.

Boggess compares two groups of patients with endometrial carcinoma: a series of 43 cases, robotically staged and a series of 101 cases laparoscopically staged. One of his major conclusions is that they "were able to perform comprehensive staging on larger women (BMI, 33 vs. 29; $p=0.008)$ " [11].

Analysis of the group of 51 patients in our work did not establish significant difference between BMI and the indices "mean operative time" $(p=0.49)$, "mean time of total pelvic lymph node dissection" $(p=0.63)$ and "EBL" $(p=0.211)$, which indirectly supports the thesis that robotically assisted hysterectomies may be equally effective as in the patients with low, so as in patients with high BMI.

\section{Conclusion}

Robotically assisted endoscopic gynecologic surgery is a modern and advanced method for operative treatment of benign and malignant gynecological tumors. It is appropriate for obese patients as the obesity is not related to a prolonged operative time.
Conflict of interest There is no actual or potential conflict of interest in relation to this article.

\section{References}

1. Seamon LG, Cohn DE, Sq V et al (2008) Robotic hysterectomy and lymphadenectomy for endometrial cancer: technical aspects and details of success-the Ohio State University method. J Robotic Surg 2:71-76

2. Sroga J, Patel SD (2008) Robotic applications in reproductive endocrinology and infertility. J Robotic Surg 2:3-10

3. Degueldre M, Vandromme J, Huong PT et al (2000) Robotically assisted laparoscopic microsurgical tubal reanastomosis: a feasibility study. Fertil Steril 74:1020-1023

4. Diaz-Arrastia C, Jurnalov C, Gomez G et al (2002) Laparoscopic hysterectomy using a computer-enhanced surgical robot. Surg Endosc 16:1271-1273

5. Senapati S, Advincula AP (2007) Surgical techniques: robotassisted laparoscopic myomectomy with the da Vinci ${ }^{\circledR}$ surgical system. J Robotic Surg 1:69-74

6. Sert BM, Abeler VM (2006) Robotic-assisted laparoscopic radical hysterectomy (Piver type III) with pelvic node dissection-case report. Eur J Gynaecol Oncol 27:531-533

7. Piver MS, Rutledge F, Smith JP (1974) Five classes of extended hysterectomy for women with cervical cancer. Obstet Gynecol 44:265-272

8. Marchal F, Rauch P, Vandromme J et al (2005) Teleroboticassisted laparoscopic hysterectomy for benign and oncologic pathologies. Surg Endosc 19:826-831

9. Magrina JF, Zanagnolo VL (2008) Robotic Surgery for Cervical Cancer. Yonsei Med J 49:879-885

10. Boggess JF, Gehrig PA, Cantrell L et al (2008) A case-control study of robot-assisted type III radical hysterectomy with pelvic lymph node dissection compared with open radical hysterectomy. Am J Obstet Gynecol 199-357:e1-e7

11. Boggess JF (2007) Robotic surgery in gynecologic oncology: evolution of a new surgical paradigm. J Robotic Surg 1:33-37 